
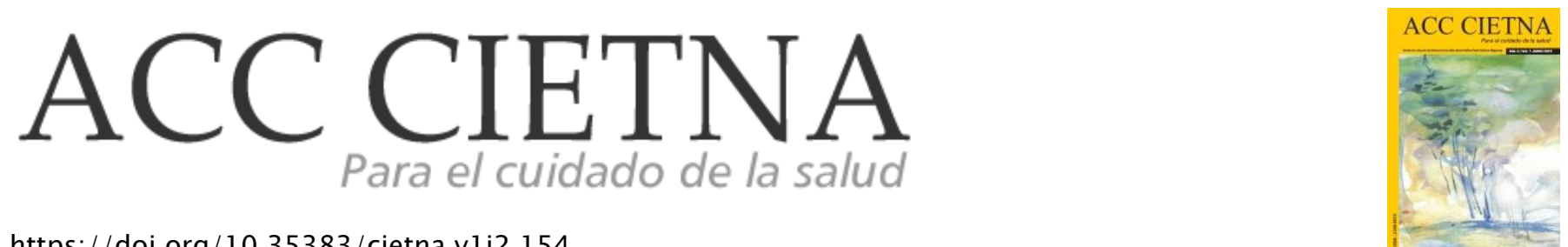

https://doi.org/10.35383/cietna.v1 i2.154

\title{
El lenguaje corporal en el cuidado a la persona hospitalizada con cáncer en fase terminal y su familiar acompañante
}

\author{
Gil Acedo Katerin Isolina ${ }^{1}$, Díaz Manchay Rosa Jeuna ${ }^{2}$
}

\begin{tabular}{l} 
INFORMACIÓN DEL ARTÍCULO \\
Historia del artículo: \\
Recibido el 10 de mayo de 2014 \\
Aceptado el 15 de agosto de 2014 \\
\hline
\end{tabular}

\section{Palabras claves:}

Lenguaje corporal

Cuidado

Enfermería

Familiar acompañante

Persona hospitalizada

\section{RESUMEN}

La presente investigación cualitativa descriptiva tuvo como objetivos: analizar, describir y comprender el lenguaje corporal en el cuidado de enfermería a la persona hospitalizada con cáncer en fase terminal y su familiar acompañante, en el servicio de oncología del Hospital Nacional Almanzor Aguinaga Asenjo, 2013. La muestra fue no probabilística, determinada por saturación y redundancia, entre los informantes tenemos seis enfermeras que laboran en el servicio de oncología, seis personas adultas con cáncer en fase terminal hospitalizadas y seis familiares acompañantes. Para la recolección de los datos se utilizó la entrevista semiestructurada. Durante la investigación se tuvieron en cuenta los criterios científicos y los principios de la bioética personalista. Se usó el análisis de contenido para el tratamiento de los datos, obteniéndose como resultados dos categorías: 1. Percepción del cuerpo ante la enfermedad en fase terminal. 2. Diálogo de presencias entre enfermera, persona hospitalizada con cáncer en fase terminal y familiar acompañante: comunicación, ternura, amabilidad, sensibilidad. La consideración final es que el lenguaje corporal en el cuidado hospitalario, incluye la percepción que tiene el enfermo sobre su cuerpo que sufre por el diagnóstico inminente de muerte, siendo indispensable el fomento de la fe, la esperanza y la paz espiritual, a través del diálogo de presencias entre enfermera, familiar acompañante y persona con cáncer en fase terminal, basado en el conocimiento del "otro", comprensión, amabilidad, toque terapéutico, carisma, servicio y caricia, de tal manera que se brinde un cuidado con amor, con sensibilidad. La

\footnotetext{
${ }^{1}$ Licenciada en Enfermería. Enfermera con trabajo independiente, Chiclayo, Perú. Email: kgil@gmail.com

${ }^{2}$ Doctora en Ciencias de Enfermería. Universidad Nacional de Trujillo. Especialista en Salud Familiar y Comunitaria por la USAT. Docente de investigación y Asesora de tesis de postgrado en Maestría en Enfermería y Maestría en Bioética y Biojuridica. Coordinadora de II Edición Maestría en Enfermería de la Escuela de Postgrado - USAT, 2010-2012, Chiclayo, Perú. Email: rdiaz@usat.edu.pe
} 
enfermera ante esta situación debería mostrarse científica, humana, sencilla y compasiva.

Body language in the care of hospitalized person with terminal cancer and their family caregivers

\section{ABSTRACT}

Keywords:

Body language

Nursing

Nurses

Family caregivers

Hospitalized person
This descriptive qualitative study aims to: analyze, describe and understand body language in nursing care to the hospitalized patient with terminal cancer and their family caregivers in the oncology department of Almanzor Aguinaga Asenjo, 2013 National Hospital. The sample was not random, determined by saturation and redundancy, being informants six nurses who work in the service of Oncology six adults hospitalized with cancer in terminal phase, and six accompanying relatives. To collect the data semiestructurada interview was used. During the investigation took into account scientific criteria and principles of the personalistic bioethics. 1: content analysis, yielding results in two categories was used. Perception of the body to the terminal illness. 2. Dialogue between nurse presences, hospitalized with terminal cancer and accompanying person familiar stage: communication, tenderness, kindness, sensitivity. The final consideration is that body language in the hospital care, including the patient perception of your body that suffers for the diagnosis of impending death, being necessary to promote faith, hope and peace of mind through the presences dialogue between nurse, family caregivers and people with cancer in terminal phase, based on knowledge of the "other ", understanding, kindness, therapeutic touch , charisma, service and caress so lovingly care that is provided, is mean sensitivity. Then the nurse should show scientific, human, simple and compassionate.

\section{Introducción}

En el actual cambio de paradigma en enfermería, se anhela suministrar un cuidado humanizado, integral y personalizado, más aún cuando la pacientes que padecen un diagnóstico mortal, como lo es el cáncer. Este cuidado es la razón de ser de enfermería y debe darse de manera horizontal entre enfermera, persona hospitalizada y familiar acompañante, de tal manera que interactúen haciendo uso de su cuerpo y corporalidad: este cuerpo que es sentido y siente, que toca y es tocado.
Al respecto, los profesionales de enfermería deben comprender las razones por las cuales el cuerpo se percibe como un objeto, con el ánimo de orientar al personal a su cargo y planear los cuidados que se requieren para ayudar a la persona. Es fundamental que los profesionales valoren la percepción que tiene la persona de su cuerpo para planear el cuidado.' Específicamente en la hospitalización de la persona con cáncer en fase terminal, la enfermera se muestra sensible y empática por la inminente muerte que será un acontecimiento esperado/inesperado por la familia, y aprovecha los momentos de cuidado 
como administración de medicamentos, ronda de enfermería, y otros procedimientos para que se relacionen e interactúen con los familiares. ${ }^{2}$

La persona con cáncer en fase terminal se ve inmersa en una familia y busca afectividad, amor, cariño, comprensión, solidaridad, respeto y cuidado; la familia contribuye a los cuidados de la persona enferma, y por esa razón debe recibir la atención e instrucción necesaria por parte del equipo de salud en especial de la enfermera para influir positivamente en la evolución de la persona enferma como del familiar.

La hospitalización de la persona con cáncer terminal genera cambios en sus estilos de vida, pues sus costumbres tendrán que adaptarse a las reglas establecidas en el hospital y ahora deberá interactuar con el personal de salud y con su familiar acompañante. Así mismo, le genera dificultad para afrontar la enfermedad terminal y aceptar la inminente muerte, piensa en los objetivos no cumplidos y valoran los aspectos básicos de la vida comer, beber, no sentir dolor. A veces esta enfermedad hace que encuentren sentido a su vida/muerte, cuando reconocen que hay un Ser Superior, que es Dios. A estos pacientes la enfermera debe acogerlos con amor y brindarles seguridad, acudir inmediatamente a su llamado ante cualquier angustia, duda o simplemente porque la persona desea abrigarse de comprensión, cariño y compasión ya que se encuentra en un medio desconocido y lejos de su familia, aunque junto al familiar acompañante.

La investigadora en su formación de pregrado ha observado que en el servicio de oncología del Hospital Almanzor Aguinaga Asenjo (H.N.A.A.A), solo algunas enfermeras al brindar cuidados a la persona con cáncer en fase terminal y su familiar acompañante, mantienen con ellos contacto físico que se manifiesta a través de un toque en el hombro, una sonrisa, una caricia, un apretón de manos, una palabra de aliento; aspectos que a veces son dejados de lado por otras enfermeras, pues predomina el cuidado biologista, como: la administración de quimioterapias, analgesia, etc. unido a la sobrecarga laboral por el excesivo número de personas hospitalizadas, entre otros aspectos administrativos.

Por lo mencionado anteriormente, surgió el siguiente problema de investigación: ¿cómo es el lenguaje corporal en el cuidado de enfermería a la persona hospitalizada con cáncer en fase terminal y su familiar acompañante, en el servicio de oncología del Hospital Nacional Almanzor Aguinaga Asenjo, 2013 ? Se determinó como objeto de estudio: El lenguaje corporal en el cuidado de enfermería a la persona hospitalizada con cáncer en fase terminal y su familiar acompañante, y como objetivos de investigación: describir y comprender el lenguaje corporal en el cuidado de enfermería a la persona hospitalizada con cáncer en fase terminal y su familiar acompañante, en el servicio de oncología de dicho hospital.

La investigación se justificó porque la enfermedad del cáncer es un problema de salud pública a nivel mundial, así lo indican las altas tasas de incidencia y mortalidad. Además existen pocos trabajos relativos al estudio del lenguaje corporal entre enfermera, familiar y persona con cáncer, por lo que la presente investigación permitió una reflexión sobre el tema desde la perspectiva de los sujetos involucrados en el cuidado de la persona con cáncer terminal, la que muchas veces es privada de un cuidado impregnado de tacto, contacto, diálogo, escucha activa, amistad, sonrisas, compasión, empatía, interés, preocupación y sensibilidad. También a través de este estudio se propició un momento de reflexión y sensibilización con las enfermeras que participaron del estudio de caso, para brindar un cuidado humanizado, basado en el respeto a la dignidad, en la equidad, la calidad y la calidez humana; considerando que el cuerpo habla a través de sus movimientos, miradas y lenguaje, haciendo conciencia de ello para poder establecer un cuidado con sensibilidad y estética, un cuidado transpersonal capaz de transformar vidas $y$ trascender. 


\section{Metodología}

Según la naturaleza de la información, la investigación fue cualitativa con abordaje descriptivo. Este tipo de investigación se define como el proceso de reflexión y análisis de la realidad a través de la utilización de métodos y técnicas para la comprensión detallada del objeto de estudio en su contexto histórico y así mismo su estructuración; observando a la persona en su vida cotidiana, obteniendo un conocimiento directo de la vida social. ${ }^{3}$ El abordaje metodológico fue descriptivo, este estudio exigió al investigador realizar una serie de informaciones sobre el objeto de estudio, luego describirlas para pasar a registrarlas, analizarlas e interpretarlas, de acuerdo a su naturaleza y los diferentes fenómenos encontrados. El objetivo es llegar a conocer las situaciones, costumbres y actitudes predominantes a través de la descripción exacta de las actividades, objetos, procesos y personas, ${ }^{4}$ de tal manera que le permitan a la investigadora analizar, describir y comprender el lenguaje corporal en el cuidado de enfermería a la persona hospitalizada con cáncer en fase terminal y su familiar acompañante.

El escenario de la investigación fue el servicio de Oncología del Hospital Nacional Almanzor Aguinaga Asenjo, en este servicio se brindan cuidados a todas las personas, desde niños hasta adultos mayores, con diagnóstico de cáncer. Tiene una capacidad de 16 camas según áreas: Reumatología y Dermatología, 2 camas; Hematología, 9 camas; y Endocrinología, 3 camas.

Participaron en la investigación 6 enfermeras que laboran en el servicio de Oncología, 6 personas adultas hospitalizadas con cáncer en fase terminal y 06 familiares acompañantes determinados por saturación y redundancia. La muestra fue no probabilística: un total de 18 sujetos de investigación.

Para guardar la confidencialidad e identidad de las participantes, se registraron a través de códigos, por orden de entrevistados. Para las enfermeras se utilizó el siguiente código: E1, E2, E3, E4, E5 y E6. Para las personas hospitalizadas con cáncer en fase terminal: P1, P2, P3, P4, P5 y P6. Para los familiares acompañantes: F1, F2, F3, F4, F5, F6. Se tuvieron en cuenta los siguientes criterios de inclusión: 1) Que la enfermera tenga como mínimo 6 meses laborando en el servicio de Oncología, 2) que la persona hospitalizada sea mayor de 18 años y tenga como diagnóstico cáncer en fase terminal, 3) que el familiar acompañante sea mayor de 18 años y no sufra de alguna enfermedad mental incapacitante.

La recolección de datos fue mediante la entrevista semiestructurada, por ser más íntima, flexible y abierta. La misma que se define como una reunión de intercambio de información entre una persona y otra. ${ }^{5}$ Se diseñó de acuerdo a lo que se quiso indagar; en este caso el lenguaje corporal en el cuidado a la persona hospitalizada con cáncer en fase terminal y su familiar acompañante. Cabe mencionar, que se realizó la validación de la entrevista con dos enfermeras nuevas del servicio de Oncología, quienes no formaron parte de la muestra, esto permitió modificar e incrementar algunas preguntas para la mejor comprensión del grupo a investigar.

Después de la aprobación del Comité de Ética en Investigación de la Facultad de Medicina de la Universidad Católica Santo Toribio de Mogrovejo, se solicitó permiso a las autoridades del Hospital Almanzor Aguinaga Asenjo y posteriormente al jefe del servicio de Oncología. Luego se procedió a identificar a los sujetos que cumplieron con los criterios de inclusión y se les presentó el consentimiento informado $y$, previa firma, se realizó la entrevista semiestructurada a las enfermeras, personas con cáncer en fase terminal y su familiar acompañante. En todo este proceso se mostró confianza e interés. Las entrevistas fueron grabadas en cintas magnetofónicas, luego se transcribieron para proceder al análisis de datos. Los datos se recolectaron entre marzo y junio de 2013. 
El análisis de datos se procesó según las fases de análisis de contenido: preanálisis, formación del sistema categorial, codificación y análisis e interpretación. ${ }^{6}$ La ética de la investigación científica estuvo basada en los principios personalistas, 7 los mismos que expresan respeto a la persona y dignidad de los participantes, responsabilidad y libertad con el consentimiento informado. De esta manera los entrevistados decidieron de manera libre participar en la investigación dando su aprobación y mostrando total disponibilidad.

Como criterios de rigor científico se consideraron: la credibilidad, la confirmabilidad y la transferibilidad, aspectos que tienen como fin aumentar la calidad de la información. ${ }^{8}$ La credibilidad, todos los datos fueron fidedignos y para dar una mejor validez a la investigación se realizó el proceso de triangulación, se consiguió tener una relación empática con las personas con cáncer en fase terminal, enfermeras y familiar acompañante, esta interrelación positiva permitió obtener datos reales. Para cumplir con la auditabilidad o confirmabilidad, se usaron grabaciones de las entrevistas, asimismo las fuentes teóricas consultadas tienen sustento científico, lo que le otorga mayor soporte y autenticidad a la investigación. Por, último la transferibilidad o aplicabilidad, todo el trabajo fue realizado con altura científica, buscando la transferencia hacia poblaciones con características semejantes a esta investigación.

\section{Resultados, análisis y discusión}

El lenguaje corporal a la persona hospitalizada con cáncer en fase terminal es abordado mediante la percepción: que siente y piensa sobre su propio cuerpo. El enfermo terminal atraviesa por un proceso de aceptación de la enfermedad creando un mecanismo de defensa que se manifiesta a través del rechazo e ira. Las enfermeras utilizan en el cuidado el lenguaje corporal, para ayudar a los enfermos a aceptar su enfermedad, esta se manifiesta a través de expresiones corporales mediadas por un diálogo de presencias, que es el encuentro entre seres humanos (enfermera/persona con cáncer terminal y familiar acompañante) que se disponen a hablar, a mirarse a los ojos, a aceptarse y enriquecerse mutuamente, esto incluye una comunicación que va de la mano de una relación amical para poder comprender a los sujetos de cuidado. Incluye también un toque de presencias que se manifiesta por medio de la ternura y la caricia, por ende la enfermera debe ser amable y mostrar siempre una sonrisa, así logra que las personas se sientan seguras y con esperanza, todo esto solo será posible si se brinda un cuidado con amor y con sensibilidad.

A continuación las categorías:

\section{Percepción del cuerpo ante la enfermedad en fase} terminal: La percepción del cuerpo de una persona con cáncer en fase terminal demuestra como una persona siente y piensa sobre su propio cuerpo:

“...Siento que me voy a morir, eso lo sé desde que me quitaron mi último seno, solo me queda esperar la muerte, cuando me quitaron el primer seno pensé que me iba a salvar con las quimioterapias y ahora estoy tirada en esta cama esperando morir..." P6

"...Es triste porque si te das cuenta que no has hecho nada en esta vida y encima que te vas a morir, yo me arrepiento de no haber tenido hijos porque no sé lo que significa ser padre, ya ni quiero verme en el espejo porque me veo pelado y flaco, quien te va a querer así, más bien siento que doy miedo..." $P 4$

“...Es un dolor muy grande tener esta enfermedad, valoras muchas cosas en este momento como por ejemplo poder tomar un poquito de agua sin ningún dolor..." P3

Se puede evidenciar que algunos aceptan que van a morir, tienen la esperanza de vivir y recuperarse; realizan un autoanálisis de cómo han vivido y la gran mayoría solo hace un recuento de las cosas 
que no han logrado, sueños truncados que no realizaron. Las personas se sienten impotentes, desesperanzadas y muy ansiosas:

"...No dejo que me hablen mucho porque solo quieren saber cómo me enfermado y no quiero que tengan pena. Saber que te vas a morir tan joven es feo, te amargas por todo porque tienes esto y no puedes hacer nada, morirse dejar a todos y que ellos de aquí a unos años te olviden es demasiado doloroso, a veces me dan ganas de dejar todo y quiero irme..." P2

Por su parte, las enfermeras revelan que los familiares a pesar de ser renuentes en aceptar la enfermedad conscienten la información que les brindan:

"...Hay familiares que son reacios no aceptan la enfermedad de su familiar, a veces ni te hablan, pero los familiares que ya están concientizados, esperando la voluntad de Dios colaboran más con su paciente..." EI

"...Algunos familiares no aceptan la enfermedad ni el estado de salud de su paciente, pero uno tiene que explicarles, informarles y ellos entienden..." E6

El proceso de aceptación de la enfermedad con diagnóstico terminal es una situación muy complicada, la persona crea un mecanismo de defensa que se manifiesta a través del rechazo debido a la noticia inesperada, muchos enfermos se vuelven agresivos, se aíslan, no colaboran con el tratamiento, se vuelven exigentes, están casi siempre malhumorados con el cuidador (familiarenfermera), evidenciándose sentimientos de resentimiento e ira.

Diálogo de presencias entre enfermera, persona hospitalizada con cáncer en fase terminal $y$ familiar acompañante: comunicación, ternura, amabilidad y sensibilidad. Durante la hospitalización es importante la comunicación, ya que a través de ella se puede lograr satisfacer las necesidades de los enfermos y familiares, generar confianza, dar seguridad, brindar apoyo afecto:
“...Logramos que confíen en nosotros, que cualquier duda nos pregunten. Logramos darles esa confianza con gesto, a través de una sonrisa, y de palabras como "estamos con usted", "Io apoyamos en todo momento", y sobre todo que conseguimos que nos comuniquen todo. Lo mismo con el familiar que confíe en nosotros, que nos comunique todo, porque estamos a su servicio"... E6

“...La enfermera cada vez que entra a trabajar viene y me saluda, me pregunta cómo estoy, si he dormido bien, cómo me encuentro, si me duele algo..." P3

A través de una adecuada comunicación y relación amical se puede captar lo que los pacientes y familiares acompañantes quieren manifestar y por ende darle solución a los problemas que pueden surgir durante la estancia hospitalaria. Estas relaciones interpersonales de amistad y comunicación se basan en gestos como una sonrisa, un trato amable, palabras de aliento como: "estamos con usted", "Io apoyamos en todo momento", "estamos a su servicio", entre otras; estos tipos de comunicación verbal y no verbal permiten generar confianza para que ambos sujetos de cuidado se expresen sin sentir bloqueos en el proceso de comunicación. Este diálogo incluye también un toque de presencias que se manifiestan por medio de la ternura y la caricia.

“...Yo trato de brindar un cuidado más humano, en ese pequeño momento al menos un contacto físico, ver qué necesidad tiene en ese momento. Entonces sabes lo tan valioso que pueden ser los gestos como un abrazo, un toque terapéutico, una palmadita o mirar a la persona cuando le hablas, esas cosas tan pequeñas pero que traen un gran resultado, a pesar del diagnóstico de la persona..." E3

“...Nos besan, nos saludan, cuando está a su alcance porque a veces están apuraditas, hay veces que se congestiona el servicio, pero siempre me ha tocado la oportunidad que me han 
acariciado, me han abrazado, me han consolado..." Pl

“...Las enfermeras prácticamente para mi mamá son la luz de la esperanza, porque ellas tratan de ayudarle a superar toda su enfermedad, cuando viene la enfermera se alegra porque le van a poner el suero, sus inyectables, en su rostro demuestra satisfacción y siente alegría de la amable atención que le brindan..." F2

\section{“...La saluda cuando llega amablemente y eso a mi} hija le gusta porque le hacen reír, le dicen hola mi reinita ya estoy aquí para consentirte, le dan aliento para que continúe a pesar del dolor que ella presenta, la calman..." $F 3$

Las enfermeras tratan de brindar un cuidado integral a las personas enfermas con cáncer en fase terminal, en los pequeños momentos que pueden interactuar con ellas tratan de mantener un contacto físico que se manifiesta a través de una palmadita en la mano o la espalda, una sonrisa, un abrazo; ante esto las personas responden cogiéndoles las manos y llenándolas de bendiciones. Como se sabe la comunicación no solo se da por palabras, las expresiones no verbales también son una forma de ésta, es por eso que las personas hospitalizadas asumen que al abrazarlos o tocarles la mano se les está diciendo que ellos pueden afrontar su proceso de enfermedad. Los enfermos terminales y familiares acompañantes mantienen esperanza por el tratamiento que las enfermeras les aplican, seguridad por el cuidado que les brindan, y confianza muy coloquial porque cada cosa que hacen bien. Así mismo los pacientes sienten agradecimiento, les dicen que son buenas, que tienen paciencia, ellos saben que la enfermera es la responsable de su bienestar y sienten alegres, protegidos y seguros.

“...Tenemos mucha sensibilidad, somos personas buenas, nos sentimos un apoyo para ellos. Uno hace bien su trabajo, uno se siente bien, lo hago con amor y cuando se hacen las cosas con amor, tú te sientes satisfecho, te sientes tranquila, te sientes bien, y en oncología tenemos que ser muy sensibles, muy amorosas, muy comprensivas..." El

"...Aprendes amar a seres que no son tu familia, entonces sabes lo valioso que pueden ser palabras, como: estoy contigo, cálmate, descansa..." E3

Las enfermeras deben ser siempre sensibles para poder brindar un cuidado con amor donde se preserven siempre los derechos de las personas como seres humanos. Enfermería es amor, una carrera profesional de ayuda al prójimo, a la búsqueda de su bienestar general, las enfermeras se catalogan como sensibles, personas buenas que siempre tratan de hacer bien su trabajo, un trabajo con amor y comprensión que busca calmar la angustia de los enfermos y sus familiares por enfrentarse ante la noticia de la muerte; los profesionales de enfermería aprenden a valorar la vida, a amar a sus pacientes, se sienten satisfechas cuando obtienen buenos resultados. A pesar del cansancio sienten que vale el esfuerzo hecho.

El cuidado humano involucra valores, voluntad y un compromiso. ${ }^{9}$ Es un ideal e implica la humanización en las relaciones. Es mantener, promover y desarrollar todo lo que exista y todo el potencial de vida que los seres vivos conservan. Todo cuidado representa compromiso afecto $y$ consideración para promover el bienestar del otro. Incluso es silencioso, interactivo y promueve el crecimiento. Es una acción de ayuda, es escuchar, tener tiempo para la reflexión y expresar sentimientos. El cuidado es amor por excelencia y no solo al cuerpo, también al alma, a lo afectivo, exige innovar, visualizar otras perspectivas, abrir horizontes: crear y descubrir, gestar y develar. 10,11

Definitivamente, el cuidado es un acto humano, un acto de amor, bondad, afecto y empatía que busca el bienestar integral de la otra persona. Se humaniza por medio de la corporalidad, ya que nuestro cuerpo habla a través de él, el cuidado es la razón de ser de enfermería y no deja de ser una excepción en las personas con cáncer en fase 
terminal, por lo que las enfermeras deben tener en cuenta cómo es que su cuerpo habla a través de sus movimientos, mirada y lenguaje. Es necesario hacer conciencia de ello para poder establecer un cuidado con sensibilidad y estética, transpersonal y capaz de transformar vidas.

El cuidado a la persona hospitalizada con cáncer en fase terminal se basa en buscar las opciones y objetivos de tratamiento que satisfagan sus necesidades físicas, emocionales, espirituales y prácticas personales, y que los familiares acompañantes sepan que su enfermo con cáncer terminal seguirá recibiendo atención y apoyo emocional durante la enfermedad. Los cuidados deben ser humanitarios y compasivos teniendo como único objetivo la calidad de vida y que los enfermos terminales enfrenten la última etapa de la vida con confianza y bienestar, en paz y dignamente. ${ }^{12}$

En el lenguaje corporal el cuerpo humano es una Sí misma, a esto se le puede definir como lenguaje. La realidad expresiva y comunicativa por sí misma $y$ en cuerpo humano es un todo expresivo $y$ comunicativo y esta capacidad expresiva de la corporalidad humana se denomina el lenguaje no verbal. El cuerpo es expresión e inclusive símbolo. El gozo, el llanto, la alegría, el dolor, la pena, la resignación, son expresiones humanas que a través del lenguaje del cuerpo, resultan muy expresivas y muy detectables en el plano de la comunicación interpersonal. La expresión corporal, en este sentido, pesa mucho más que la cadena verbal y en el rostro de un ser humano es posible visibilizar con mucha claridad la frustración a pesar de que él se obstine en negarla mediante palabras. ${ }^{13}$

El lenguaje corporal juega un papel muy importante ante el cuidado de una persona hospitalizada con cáncer en fase terminal, ya que son personas susceptibles enfrentadas ante el diagnóstico de la muerte, se encuentran sensibles en todas sus dimensiones y lo único que necesitan es que las enfermeras a través de su lenguaje corporal le transmitan amor mediante las miradas, los gestos y toda expresión corporal en general; entonces la enfermera deberá mostrarse humana y compasiva con el único objetivo de brindar un cuidado humanizado.

El lenguaje corporal a la persona hospitalizada con cáncer en fase terminal es abordado mediante la percepción del cuerpo, donde se enfoca la imagen corporal de las personas con cáncer en fase terminal y se demuestra como una persona siente y piensa sobre su propio cuerpo, el mismo que atraviesa por un proceso de aceptación de la enfermedad, situación complicada que hace que la persona desarrolle un mecanismo de defensa que se manifiesta a través del rechazo e ira. Por tal motivo las enfermeras deben incluir en el cuidado la corporalidad para ayudar a los enfermos con cáncer en fase terminal a aceptar su enfermedad.

En la primera categoría, Percepción del cuerpo ante la enfermedad en fase terminal, es preciso afirmar que la enfermedad altera profundamente la percepción de la propia corporalidad. En el plano médico, el cuerpo humano es un organismo complejo que tiene distintas funciones y órganos y que puede experimentar algún desorden o proceso de caotización que, generalmente, se manifiesta en la enfermedad. La experiencia de la enfermedad significa, desde el punto de vista de la corporalidad, la transición de cuerpo-amo a cuerpo-esclavo. Cuando la persona está enferma, se da cuenta que no puede regular ni dirigir su cuerpo, que no es el alma de su cuerpo, si no que ella se convierte en esclava de aquel. Por otro lado, cuando uno está sano, ni siquiera se percata de la existencia de su cuerpo, no se da cuenta de la cantidad de miembros y de partes que tiene su corporalidad. Se siente bien en su cuerpo, está completamente amoldado a él. Sin embargo, cuando la persona sufre un proceso de enfermedad, entonces padece el peso de su corporalidad, siente su cuerpo como una carga, como el peso más pesado, que hay que trasladar y cuidar para evitar males mayores. La persona enferma no es dueña de su cuerpo, si no que se ha 
convertido en su sirvienta. La vivencia de una enfermedad supone el reconocimiento de la propia fragilidad constitutiva del ser y desde este punto de vista la persona adquiere un conocimiento más adecuado de sí mismo. La enfermedad puede activar enormemente el proceso de autoconocimiento personal. El enfermo focaliza su atención en la zona del dolor, la experiencia de la corporalidad es mucho más intensa en la enfermedad que en la salud, en el sufrimiento que en el placer. ${ }^{13}$

En la presente investigación debido al diagnóstico de cáncer en fase terminal, las personas ven afectadas su corporalidad, se sienten con un malestar que les causa mucho dolor en el cuerpo, asimismo presentan alopecia y disminución del peso. Pasan por el periodo de transición del cuerpo-amo a cuerpo-esclavo; se dan cuenta que no pueden regular su cuerpo, y empiezan a valorar muchas cosas que no eran consideradas cuando estaban sanos, como por ejemplo: el tomar un vaso de agua sin ningún dolor o sucesos importantes como el tener un hijo. Se convierten en esclavas de su propio cuerpo porque se han dado cuenta que dependen de él para poder vivir; la forman en cómo viven esta enfermedad hace que reconozcan lo frágil que puede ser el cuerpo humano, en consecuencia esto hace que algunos pacientes acepten la enfermedad y otras guarden la esperanza de vivir debido al tratamiento que les brindan en el hospital. Ante esto los pacientes con cáncer en fase terminal atraviesan por fases de aceptación de la misma.

La segunda categoría, Diálogo de presencias entre enfermera, persona hospitalizada con cáncer en fase terminal y familiar acompañante: comunicación, ternura, amabilidad, sensibilidad, revela la importancia de estar presente, de ayudar, apoyar, estar alerta y pendiente; de llegar a la interioridad de la persona con cáncer en fase terminal y familiar acompañante mediante un lenguaje verbal y no verbal. 13 La enfermera con su comunicación empática ayuda a la persona hospitalizada a ver con más claridad los comportamientos motivados por sus conflictos o emociones, debe aprender a ser suficientemente acogedora, amable y neutra para que el otro pueda abrirse y expresarse como es, sin sentir bloqueos en la comunicación. ${ }^{14}$

En este trabajo se demuestra que a través de una relación amical y una comunicación empática se podría captar lo que los enfermos y familiares acompañantes nos quieren manifestar $y$, por ende, con llevaría a dar solución a los problemas que puedan surgir durante la estancia hospitalaria.

Las enfermeras comprensivas logran justificar o entender como naturales, las acciones o las emociones de otros, ya que estos son seres humanos que sienten y sufren. ${ }^{15}$ La comprensión durante el proceso de cuidado en enfermería es importante porque va a permitir establecer una relación óptima con el fin de lograr el bienestar del enfermo. Al ser comprensivos también somos empáticos, la empatía le sirve a la enfermera para que finalmente pueda comprender la situación de salud por la que está atravesando la persona con cáncer en fase terminal y su familiar acompañante. Todo esto creará un clima de confianza y ayuda mutua, idóneo para resolver las dudas existentes.

La persona con cáncer en fase terminal necesita ser acogida, estar acompañada y ser tratada con amabilidad, ser escuchada y comprendida; también sentirse útil, ser respetada y protegida. El diálogo de presencias incluye también "un toque" de presencias que se manifiesta por medio de la ternura y la caricia, esto es, una palmadita en la mano o la espalda, una sonrisa.

La enfermera que cuida a personas con cáncer en fase terminal debe ser cariñosa, afectuosa, gentil, agradable, servicial, afable, incluso graciosa, portadora de un buen humor. A través de la amabilidad las enfermeras hacen que sus pacientes se sientan queridos, alegres y parte de su vida diaria, ya que es expresión externa, es una actitud interna de ternura y bondad constante hacia otra persona. Una sonrisa en el rostro acercará más a los sujetos de cuidado, una sonrisa 
se deberá considerar entonces como el medicamento más eficaz y barato, sin efectos secundarios, ayudará a crear actitudes positivas y optimistas.

La seguridad se logra a través de la comunicación empática que busca mantener la información como primer principio. En este ambiente, podemos transmitir el valor de la esperanza, ayudar al enfermo terminal a aceptar su situación y a construir su sentido de la vida a partir de sus creencias.

\section{Conclusiones}

El lenguaje corporal de la persona hospitalizada con cáncer en fase terminal es abordado mediante la percepción del cuerpo: cómo siente y piensa sobre su propio cuerpo, que se está enfrentado al diagnóstico de finitud de la vida e inminente muerte. Ellos atraviesan por un proceso de aceptación de la enfermedad y crean un mecanismo de defensa que se manifiesta a través del rechazo e ira, siendo indispensable el fomento de la fe, la esperanza y la paz espiritual. Por eso necesitan ser respetados, acogidos, escuchados, comprendidos y tratados con amabilidad. Entonces la enfermera debería mostrarse científica, humana, sencilla y compasiva.

El lenguaje corporal en el cuidado a la persona hospitalizada con cáncer en fase terminal y su familiar acompañante incluye un diálogo de presencias, basado en la comunicación, comprensión, amabilidad, tacto, contacto y ternura para poder lograr una relación amical y empática. La enfermera podría ser carismática, afectuosa, gentil, cortés, agradable, servicial, portadora de una sonrisa y capaz de brindar una caricia como lo es una palmadita en la mano o en la espalda, las manos de la enfermera constituyendose su principal instrumento para cuidar, para brindar un cuidado con amor, es decir con sensibilidad.

\section{Bibliografía}

1. Moreno Fergusson María E. Cuerpo y Corporalidad en la Paraplejia: Una teoría de enfermería. [Tesis doctoral]. Bogotá: Universidad Nacional de Colombia. Facultad de Enfermería; 2011.

2. Díaz y Bustamante. Enfermería, familia y persona con cáncer terminal. Del cuidado hospitalario al cuidado en el hogar. Perú: Emmanuel; 2011.

3. Burns, N. Investigación en Enfermería. 3 era edición. Saunders. Madrid-España, 2005.

4. Oliveira M. Como fazer pesquisa qualitativa. Editora Vozes. Petrópolis, Rio de Janeiro; 2004.

5. Hernández Sampieri y colaboradores. Metodología de la investigación: cuantitativa y cualitativa $4^{a}$.ed. México: Mc Graw-Hill Interamericana; 2006.

6. Pérez, G. Investigación Cualitativa- Retos e interrogantes II- Técnicas y Análisis de datosEditorial: la muralla. S. A-; Madrid- 1991

7. Sgreccia E; "Manual de Bioetica". La bioética y sus principios. $4^{\circ}$ Edición. Madrid: Talisio; 2007.

8. Tello C, Gutiérrez N, Pérez C. Métodos y técnicas de análisis cualitativo. Sección de Postgrado en Enfermería-Escuela de Postgrado- Universidad Nacional de Trujillo. Trujillo; 2009.

9. Díaz Manchay Rosa y Bustamante Edquén Sebastián. Enfermería, familia y persona con cáncer terminal. Del cuidado hospitalario al cuidado en el hogar. Perú: Emmanuel; 2011.

10. Waldow R. Cuidar: Expressão humanizadora da enfermagem. Editora Vozes. Petrópolis, Rio de Janeiro; 2006. 
11. Ancízar Manuel y otros. Cuidado de la Vida: Facultad de Enfermería. Colombia. Universidad Nacional de Colombia. 2006.

12. American Society of clinical on cology. Planificación de los cuidados del enfermo con cáncer en etapa avanzada. Lo que los pacientes y sus familiares deben saber acerca de sus opciones al enfrentarse a una enfermedad grave. 2011. Estados Unidos [Acceso 21 de Septiembre de 2013]. Disponible en:

http:/ / www.cancernet/sites/cancer.net/files/vign etto/advanced_cancer_care_plannig_ESP.pdf
13. Torralba, F. Antropología del cuidar. Barcelona: Fundación Mapfre Medicina; 1998.

14. Cibanal Juan L. y Carmen Arce M. La relación enfermera - paciente. Colombia. Universidad de Antoquia. 2009.

15. Weis Bd. Health literacy: A manual for clinicians. American Medical Association. Estados Unidos. American Medical Associaton Foundation. 2003. 